\title{
Optimization of Sperm Culture Conditions for Human Assisted Reproductive Technologies
}

\author{
Carol L Curchoe ${ }^{1}$ and Charles L Bormann ${ }^{2 *}$ \\ ${ }^{1}$ San Diego Fertility Center, San Diego, USA \\ ${ }^{2}$ Harvard Medical School, Obstetrics/Gynecology/Reproductive Endocrinology and Infertility, USA
}

Submission: February 02, 2018; Published: March 23, 2018

*Corresponding author: Charles L Bormann, San Diego Fertility Center, San Diego, CA, 32 Fruit Street, Boston, MA 02114, USA, Tel: 617-724-1807; Email: cbormann@partners.org

Abstract

Optimizing sperm performance can be a differentiating factor in clinical pregnancy rates for assisted reproductive technology (ART) laboratories. Minimizing cellular stress during routine sperm processing by maintaining a precise and stable environment is of great importance in the field. In commercial media, $\mathrm{pH}$ of the external cellular culture environment ( $\mathrm{pHe}$ ) is typically maintained at 7.2-7.4 by various buffering reagents, while the internal $\mathrm{pH}$ of the cell (pHi) depends on available lactate and amino acids. Combining buffers in solution has been previously shown to be valuable for stabilizing internal and external $\mathrm{pH}$ for various biological systems. We hypothesized that a dual-buffer culture medium might improve sperm performance for ART. Here we demonstrate superior performance of a commercially available dual-buffer solution of HEPES and MOPS: Multipurpose Handling Medium (MHM, Irvine Scientific). Significantly better performance, assessed at 8 and 48 hours, and measured as: sperm viability, total motility, and rapid forward progression, was observed for MHM over single buffer controls.

Keywords : Sperm processing; Sperm wash; Sperm viability; Multipurpose handling medium

Abbreviations : ART: Assisted Reproductive Technology; MHM: Multipurpose Handling Medium; SPWASH: Sperm Washing Medium; CASA: Computer Assisted Sperm Analyzer; IQR: Inter Quartile Range; MHM: Multipurpose Handling Media; AAB: American Association of Bioanalysts

\section{Introduction}

Achieving and maintaining high pregnancy rates is a top priority for embryology labs and fertility practices. Optimizing sperm performance can be a key differentiating factor in achieving better clinical pregnancy rates. Human sperm handling procedures for ART are commonly performed under atmospheric conditions, where temperature and $\mathrm{CO}_{2}$, play a significant role in regulating $\mathrm{pH}$. Maintaining a precise and stable $\mathrm{pH}$ balance is challenging, as even minor environmental fluctuations can negatively impair human sperm function. HEPES and MOPS are zwitter ionic organic buffers with pKa at $20^{\circ} \mathrm{C}$ of $\sim 7.55$ and 7.15 , respectively. Individually, they have been extensively studied in numerous ART-related processes, including: sperm isolation, oocyte retrieval, ICSI, embryo biopsy, embryo transfer, and cryopreservation. However, recent evidence supports the notion that combining HEPES: MOPS may allow for improved media formulations.

Multipurpose Handling Medium (MHM, Irvine Scientific) is a commercially available dual-buffer solution of HEPES and MOPS used to maintain stable conditions for oocytes, and embryos when being manipulated under atmospheric conditions. Dualbuffer solutions have demonstrated improvements in working $\mathrm{pHe}, \mathrm{Na}^{+}$or $\mathrm{K}^{+}$concentration, and/or concentration toxicity and osmolality, over single buffer media, and is specially formulated to maintain a physiological $\mathrm{pH}$ of 7.2-7.4 over a broad temperature range. We hypothesize that a dual-buffer Multipurpose Handling Medium will better support and maintain sperm viability parameters compared to specimens processed with a single buffer solution.

Here we describe the baseline conditions for human semen samples in three handling media (dual buffer MHM compared to two commercially available single buffer HEPES media: a control sperm washing medium, Sperm Washing Medium (SPWASH, Irvine Scientific), and an ART handling medium, Quinn's Advantage Medium with HEPES (QUINN, Cooper Surgical), by distribution of motility percent and rapid progression percent, and we quantify causal association of motility percent and rapid progression percent to handling medium after 48 hours at roomatmosphere conditions.

\section{Materials and Methods}

\section{Sperm collection, preparation and processing}

Semen samples from 21 men undergoing routine fertility testing and having normal specimen parameters as defined by the WHO $5^{\text {th }}$ edition [1] were included in this study. Specimens 


\section{Global Journal of Reproductive Medicine}

were liquefied for 30-60 minutes and sperm were quantified using a Computer Assisted Sperm Analyzer (CASA; CEROS ${ }^{\mathrm{TM}}$ II, Hamilton Thorne). Specimens meeting eligibility criteria were layered over a 50:90\% Isolate gradient (Isolate, Irvine Scientific) and centrifuged for 20 minutes at 300xg. Following centrifugation, sperm pellets were suspended with $10 \mathrm{ml}$ of SPWASH and centrifuged for 10 minutes at $300 \mathrm{xg}$. This wash step was performed two times for each specimen. The final sperm pellet was quantified using CASA and equivalent concentrations of motile sperm $(\sim 5$ million $/ \mathrm{ml})$ were added to the following treatment groups: SPWASH, QUINN, and MHM. All specimens were analyzed under IRB approval.

\section{Data collection and statistics}

Following collection, processing, and preparation of participants semen samples in three handling media- labeled and capped tubes were left in a controlled room temperature environment for 48 hours. Assessments were performed at 8, 24, and 48 hours, at which time the concentration, motility percent, rapid motile progression percent, medium motile progression percent, slow motile progression percent, and static motile percent of total sperm were recorded. The mean and standard deviation of the recorded baseline (0-hour) characteristics were calculated to describe the observed location and spread of these variables, by handling medium and overall. The distributions of motility percent and rapid progression percent within each handling medium are summarized in box plots at baseline and each follow-up time.

The overall association between motility percent and handling medium was assessed using a linear mixed effects regression model. This model estimates the average motility percent of sperm in each medium, adjusting for the motility percent at baseline and accounting for possible correlation between measurements on the three samples taken from the same individual. Separate models were used to estimate motility percent at 8 hours and at 48 hours. Analogous linear mixed effects models were fit to assess the association between rapid progression percent and handling medium at 8 hours and at 48 hours.

\section{Results}

Semen samples from 21 individuals, and each sample was prepared following the same laboratory protocol and divided into three subsamples, one in each of the study media (MHM, QUINN, SPWASH), for a total of 63 samples. The study design is balanced across subjects and sperm handling media, and there is no missing data for the analyzed variables. The mean and standard deviation of baseline characteristics of the samples are shown in Table 1. The concentration of sperm is similar across handling media, and there were no statistical differences in total motility or motility progression categories across the three treatment groups. Box plots comparing the distributions of motility percent in MHM, QUINN, and SPWASH at baseline and at 8,24 , and 48 hours are shown in Figure 1. In these plots, the horizontal black line dividing the box indicates the median (or $50^{\text {th }}$ percentile) of motility percent for samples in the specified medium. The bottom and top of the colored box correspond to the observed $25^{\text {th }}$ percentile and $75^{\text {th }}$ percentile of motility percent values, respectively. This interval is also called the inter quartile range (IQR). The vertical lines extending from the box indicate the range of observed values within a distance of 1.5 times the length of the IQR from the median. Motility percent appears to be similarly distributed across the three media at baseline, but as time progresses, the motility percent declines more in QUINN and SPWASH than it does in MHM.

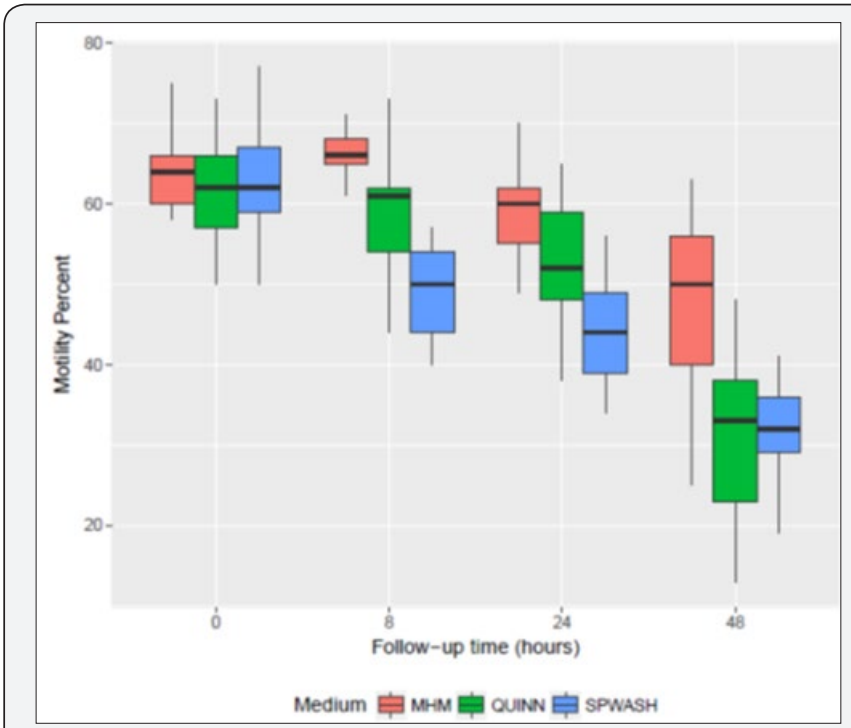

Figure 1: Distribution of motility percent, stratified by handling medium and follow-up time.

Table 1: Mean and standard deviation (SD) of baseline (0-hour) conditions, overall and by medium type.

\begin{tabular}{|c|c|c|c|c|}
\hline Mean (SD) & MHM & QUINN & SPWASH & Overall \\
\hline $\begin{array}{c}\text { Concentration } \\
(\mathrm{mi} / \mathrm{ml})\end{array}$ & $5.03(0.23)$ & $5.09(0.28)$ & $5.01(0.26)$ & $5.04(0.26)$ \\
\hline Motility (\%) & $\begin{array}{c}63.38 \\
(5.87)\end{array}$ & $\begin{array}{c}61.14 \\
(7.80)\end{array}$ & $\begin{array}{c}62.10 \\
(7.73)\end{array}$ & $\begin{array}{c}62.21 \\
(7.13)\end{array}$ \\
\hline \multicolumn{5}{|c|}{ Progression (\%) } \\
\hline Rapid & $\begin{array}{c}48.33 \\
(13.81)\end{array}$ & $\begin{array}{c}47.81 \\
(12.41)\end{array}$ & $\begin{array}{c}49.00 \\
(7.06)\end{array}$ & $\begin{array}{c}48.38 \\
(11.29)\end{array}$ \\
\hline Medium & $8.95(7.84)$ & $5.71(4.56)$ & $7.14(5.62)$ & $7.27(6.21)$ \\
\hline Slow & $6.10(7.66)$ & $7.62(9.04)$ & $5.95(5.85)$ & $6.56(7.55)$ \\
\hline Static & 36.62 & 38.86 & 37.90 & 37.79 \\
$(7.87)$ & $(7.80)$ & $(7.73)$ & $(7.13)$ \\
\hline
\end{tabular}

The estimated differences in mean motility percent at 8 hours and at 48 hours, comparing samples of sperm preserved in QUINN to those in MHM and comparing samples preserved in SPWASH to those in MHM are shown in Table 2. Each estimate is accompanied by a $95 \%$ confidence interval for the true difference between mediums as well as a p value quantifying the strength of evidence in the data supporting the difference. For example, at 8 hours, the mean motility percent in QUINN is estimated to be 7.12 lower than the mean motility percent in MHM, comparing 


\section{Global Journal of Reproductive Medicine}

samples with the same baseline motility percent. We are $95 \%$ confident that the true difference in motility percent between the mediums (QUINN minus MHM) at 8 hours is within the interval -10.57 to -3.68 . The $\mathrm{p}$ value for the statistical test that the true difference is nonzero is $p=1: 62 \times 10^{-4}$ : This value estimates the probability that a difference between mediums of -7.12 would be observed if indeed there were no difference in motility percent between the mediums, and the difference in observed values were only due to random chance. A very small $p$ value indicates that the data contain strong evidence that the mean motility percent after 8 hours in QUINN is different from, and in this case lower than, that in MHM. Typically, a p value of less than 0.05 is interpreted as a statistically significant association. The values in the table for the difference in motility percent between QUINN and MHM at 48 hours and for the difference in mean motility percent between SPWASH and MHM at 8 hours and at 48 hours may be interpreted analogously.

Table 2: Estimated difference in mean motility percent of sperm preserved in QUINN compared to MHM, and in SPWASH compared to MHM, at 8 hours and at 48 hours.

\begin{tabular}{|c|c|c|c|}
\hline Medium & Estimate & 95\% CI & p Value \\
\hline \multicolumn{4}{|c|}{ QUINN } \\
\hline $8 \mathrm{H}$ & -7.12 & $(-10.57,-3.68)$ & 0.000162 \\
\hline $48 \mathrm{H}$ & -17.04 & $(-21.65,-12.44)$ & $3.38 \mathrm{E}-09$ \\
\hline \multicolumn{4}{|c|}{ SPWASH } \\
\hline $8 \mathrm{H}$ & -16.6 & $(-20.04,-13.18)$ & $2.71 \mathrm{E}-12$ \\
\hline $48 \mathrm{H}$ & -17.14 & $(-21.71,-12.57)$ & $2.58 \mathrm{E}-09$ \\
\hline
\end{tabular}

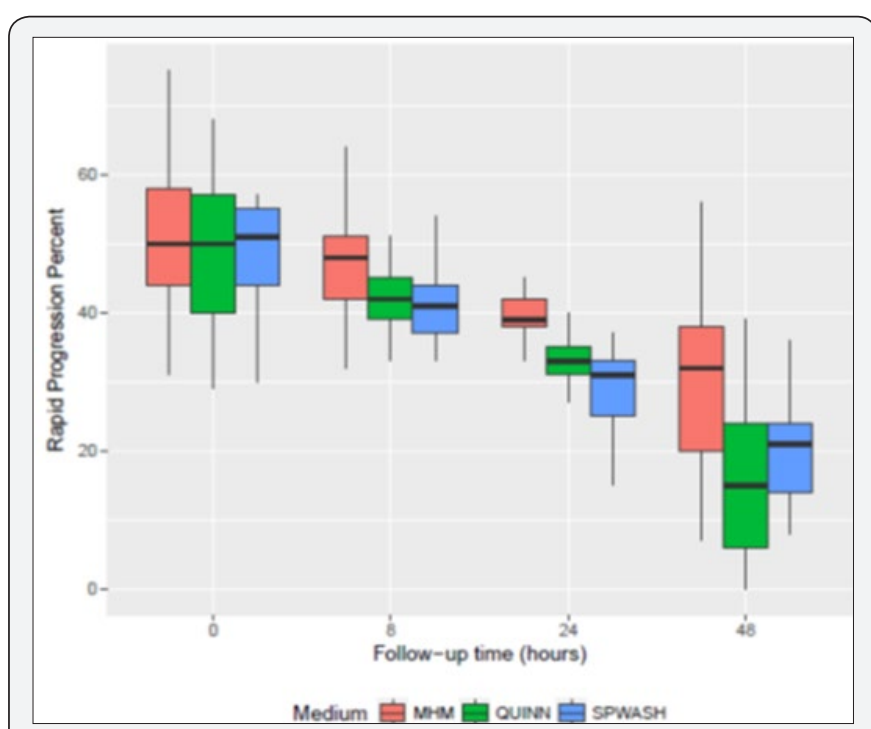

Figure 2 : Distribution of rapid progression percent, stratified by handling medium and follow-up time.

For example, at 48 hours, the mean motility percent in SPWASH is estimated to be 17.14 lower than the mean motility percent in MHM, comparing samples with the same baseline motility percent, with a $95 \%$ confidence interval of $(-21.71$, -12.57) for the true difference and a $p$ value $p=2: 58 \times 10^{-9}$ : Box plots comparing the distributions of rapid progression percent in MHM, QUINN, and SPWASH at baseline and at 8, 24, and 48 hours are shown in Figure 2. As was observed for overall motility percent, the rapid progression percent appears to be similarly distributed for each of the three media at baseline. At each subsequent follow-up time, the rapid progression percent appears to decline more in QUINN and SPWASH than it does in MHM.

Table 3: Estimated difference in mean rapid progression percent of sperm preserved in QUINN compared to MHM, and in SPWASH compared to MHM, at 8 hours and at 48 hours.

\begin{tabular}{|c|c|c|c|}
\hline Medium & Estimate & $\mathbf{9 5 \%}$ CI & p Value \\
\hline \multicolumn{4}{|c|}{ QUINN } \\
\hline $8 \mathrm{H}$ & -5.2 & $(-9.39,-1.00)$ & 0.017 \\
\hline $48 \mathrm{H}$ & -13.16 & $(-18.71,-7.09)$ & $8.53 \mathrm{E}-05$ \\
\hline \multicolumn{4}{|c|}{ SPWASH } \\
\hline $8 \mathrm{H}$ & -8.21 & $(-12.40,-4.02)$ & 0.000321 \\
\hline $48 \mathrm{H}$ & -10.6 & $(-16.09,-5.04)$ & 0.0011 \\
\hline
\end{tabular}

The estimated differences in mean rapid progression percent at 8 hours and at 48 hours, comparing samples of sperm preserved in QUINN to those in MHM and comparing samples preserved in SPWASH to those in MHM are shown in Table 3. Each estimate is accompanied by a $95 \%$ confidence interval for the true difference between mediums and a $\mathrm{p}$ value quantifying the strength of evidence in the data supporting the difference. The interpretations of the results of the linear regression in Table 3 are analogous to those shown in Table 2. For example, at 48 hours, the mean rapid progression percent in QUINN is estimated to be 17.04 lower than that in MHM, comparing samples with the same baseline rapid progression percent. The 95\% confidence interval for the true difference in rapid progression percent between QUINN and MHM is (-21.65, $-12.44)$. The $p$ value associated with this estimate is $p=3: 38 x$ $10^{-9}$, which indicates very strong evidence in the data supporting the difference in mean rapid progression in QUINN and MHM. As for motility percent above, differences of substantial magnitude and strong statistical significance were observed for rapid progression percent at 8 hours and at 48 hours, between QUINN and MHM and between SPWASH and MHM.

\section{Discussion}

Replicating physiological homeostasis minimizes cellular stress during routine sperm handling, generating optimal outcomes for assisted reproductive procedures, such as: IUI, ICSI, and IVF [2]. During insemination, gametes and embryos are readily maintained at ideal temperature, humidity, and $\mathrm{CO}_{2}$ levels. The $\mathrm{CO}_{2}$ gas concentration regulates external $\mathrm{pH}$. pHe is easily manipulated and measured; therefore, optimal pHe has been extensively examined for embryo and gamete culture. However, handling, processing, and preparation steps are typically performed outside of an incubator, where sperm are exposed to atmospheric conditions, and the risk of even minor changes in pHe can lead to undesirable outcomes in sperm viability and performance, and by extension fertility outcomes [3]. 
Zwitter ionic buffers, such as HEPES and MOPS, can act as either an acid or a base and are commonly used to stabilize $\mathrm{pHe}$ in cell culture media. It has been shown that pHe influences cells in culture through its impact on intracellular $\mathrm{pH}(\mathrm{pHi} ; 4,5)$. pHi in turn, regulates a variety of cellular processes including enzymatic activity, cell division, differentiation, membrane transport, protein synthesis, cell communication, cytoskeleton elements and microtubule dynamics [3]. Cells contain intrinsic pHi regulatory mechanisms, $\left(\mathrm{HCO}_{3}^{-} / \mathrm{Cl}-\right.$ exchanger, $\mathrm{Na}^{+} / \mathrm{H}^{+}$ antiporter, and $\mathrm{Na}^{+}$dependent $\mathrm{HCO}_{3}-\mathrm{Cl}$ - exchanger); however, in culture, pHi initially follows the external $\mathrm{pH}$ of media. For clinical use, buffers should be stable, easily prepared, and consistent lot-to-lot. HEPES and MOPS can be obtained as a free acid, or conjugated to various salts; like sodium and potassium. Combining free acid and salt forms of the same buffer in various ratios offers the ability to adjust the working pHe of the medium during formulation, without the need to adjust with an acid or base later, improving consistency of the media formulation [4].

Multipurpose Handling Media (MHM, Irvine Scientific) combines HEPES and MOPS for an improved formulation that does not require the use of a $\mathrm{CO}_{2}$ incubator to maintain physiological $\mathrm{pH}$ 7.2-7.4 and osmolality over a broad temperature range. Additionally, MHM solution also contains glycine and taurine to maintain cellular homeostasis through regulation of pHi [5]. Combining HEPES and MOPS for embryo culture demonstrated that the use of both buffers provide buffering at a point between HEPES or MOPS alone, and yielded similar blastocyst formation and cell number compared to the individual buffers [6]. Furthermore, preliminary studies suggest that a combination buffer of HEPES/MOPS may be useful for procedures such as ICSI, as it yielded similar rates of normal fertilization of human oocytes (71\%) abnormal fertilization (5\%) and blastocyst development $(74 \%)$ as media buffered with HEPES alone $(63 \%$, $12 \%, 52 \%$, respectively; 6).

Human sperm bioassays are highly sensitive when both motility and the quality of sperm motility (motility grade) are taken into consideration [7]. The American Association of Bioanalysts $(\mathrm{AAB})$ chose an assay time of 48 hours for identifying the quality of ART culture media, we used 48 hours as an endpoint to be consistent with this guidance, and assayed motility percent and rapid progression percent, to sensitively quantify causal association of sperm performance to handling medium. The experimental design of this study enabled the causal association between handling medium and rapid and total sperm motility percent. However, it is important to carefully assess whether any unmeasured factors or factors not analyzed could explain part or all of the observed differences in motility percent and rapid progression percent between the handling media. Precise handling of cells and supplies within the lab, as well as tedious attention to detail are just as important as the media formulation to outcomes. We took care to minimize any differences across media in the handling of samples throughout the experiment and in the measurement tools or procedures used at baseline or during follow-up, so as not to introduce bias into the data.

\section{Conclusion}

Differences of substantial magnitude and strong statistical significance were observed for mean motility percent between QUINN and MHM, and between SPWASH and MHM, at both the analyzed follow-up times. Here for the first time, we have demonstrated superior performance of a commercially available dual buffer solution of HEPES and MOPS for human sperm handling. Sperm viability, motility percent, and rapid progression in MHM displayed significantly better performance than single buffer controls, presumably due to synergistic pHe and pHi stabilization afforded by a dual-buffered system.

\section{References}

1. World Health Organization (2010) WHO Laboratory Manual for the Examination and Processing of Human Semen $\left(5^{\text {th }}\right.$ Edn $)$. World Health Organization; Geneva, Switzerland.

2. Simopoulou M, Gkoles L, Bakas P, Giannelou P, Kalampokas T, et al. (2016) Improving ICSI: A review from the spermatozoon perspective. Syst Biol Reprod Med 62(6): 359-371.

3. Will MA, Clark NA, Swain JE (2011) Biological pH buffers in IVF: help or hindrance to success. J Assist Reprod Genet 28(8): 711-724.

4. Regula CS, Pfeiffer JR, Berlin RD (1981) Microtubule assembly and disassembly at alkaline pH. J Cell Biol 89(1): 45-53.

5. Boron WF (1986) Intracellular pH regulation in epithelial cells. Annu Rev Physiol 262(1Pt 1): L1-14.

6. Swain JE, Pool TB(2009) New pH-buffering system for media utilized during gamete and embryo manipulations for assisted reproduction. Reprod Biomed Online 18(6): 799-810.

7. Hossain A, Aryal S, Osuampke C, Phelps J (2010) Human sperm bioassay for reprotoxicity testing in embryo culture media: some practical considerations in reducing the assay time Adv Urol 10: 136898. 


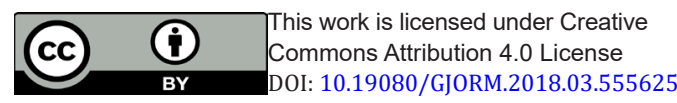

\begin{tabular}{|l|}
\hline \multicolumn{1}{|c|}{ Your next submission with Juniper Publishers } \\
will reach you the below assets \\
- Quality Editorial service \\
- Swift Peer Review \\
- Reprints availability \\
- E-prints Service \\
- Manuscript Podcast for convenient understanding \\
- Global attainment for your research \\
- Manuscript accessibility in different formats \\
( Pdf, E-pub, Full Text, Audio) \\
- Unceasing customer service \\
Track the below URL for one-step submission \\
https://juniperpublishers.com/online-submission.php
\end{tabular}

